Polymer Journal, Vol. 7, No. 2, pp 172-180 (1975)

\title{
A New Thermo-Dielectric Loss Measurement for Estimating Polymer Compatibility
}

\author{
Saburo Akiyama, Yoshinobu Komatsu, and Rokuro Kaneko \\ Department of Industrial Chemistry, Faculty of Technology, Tokyo University of \\ Agriculture and Technology, Koganei, Tokyo, Japan.
}

(Received April 8, 1974)

\begin{abstract}
The thermo-dielectric loss measurement is a technique whereby the change of dielectric loss is measured under a definite temperature program. In this method, dielectric loss is determined by the DTA method, since dielectric loss is finally transformed to heat. The contour maps of $\operatorname{loss} \sim \log f \sim 1 / T$ are easily drawn from the DTA curves of polymers and their mixtures.

The results obtained from these maps provide new and interesting information about the estimation of polymer compatibility. The compatible pairs poly(vinyl nitrate)(PVN)poly(vinyl acetate) (PVAc) and PVN-ethylene vinyl acetate copolymer (86-wt $\%$ vinyl acetate) (EVAc) are quite different from the incompatible pair PVAc-EVAc in the topographical shape of these contour maps. In these maps, it was found that the maps of compatible and incompatible mixture corresponded well to the maps of random copolymer and of graft copolymer, respectively. And judging by the sharpness of the temperature and frequency dispersion curves of normalized dielectric loss, it was found that even among these compatible pairs, the PVN-PVAc mixture could be presumed more homogeneous than PVN-EVAc mixture.
\end{abstract}

KEY WORDS Thermo-Dielectric Loss / Polymer Compatibility / DTA / Contour Maps / Vinyl Polymers / Random Copolymer / Graft Copolymer / Activation Energies / Transition Temperatures /

Dielectric relaxation measurement is one of the most useful methods for finding information about the relationship between the mobility of polar polymer chains and their chemical structures or states of aggregation. The measurements of temperature dispersion or frequency dispersion of dielectric loss are actually indispensable in order to study the mechanism of relaxation and the distribution of relaxation times. And the application of this method for studying fine structures of amorphous polymers has recently increased remarkably. But a simplification of the apparatus is still required to make further applications of this dielectric method. The DTA technique reported here will supply this requirement. Matsuo, Suga, and Seki ${ }^{1}$ have adopted the DTA technique to chemical analysis and showed how simple it was to use thermal analysis to study the dielectric properties. Amaya ${ }^{2}$ also tried to make an apparatus to investigate dielectric and magnetic properties with the DTA technique.
Our apparatus was made to be applied for solid polymer samples. Since the outline of this work have already been reviewed ${ }^{3}$, the present paper is intended to offer a detailed explanation of the apparatus and one of its interesting application which is to estimate the polymer compatibility.

Using various methods, Poly(vinyl nitrate) (PVN) - poly(vinyl acetate) (PVAc) and PVNethylene-vinyl acetate copolymer $(86.0 \mathrm{wt} \%$ vinyl acetate) (EVAc) have been determined to be compatible pairs $^{4-6}$, and the PVAc-EVAc mixture to be an incompatible pairs ${ }^{6}$. A random copolymer, poly(vinyl chloride-co-vinyl acetate), poly(VC-VAc), and a graft copolymer composed of poly(vinyl chloride) (PVC) and EVAc, (PVC-EVAc) graft copolymer, were also measured to compare their dielectric behavior with the above mixture.

\section{THEORETICAL}

When polar polymers are put in an alternating 
electric field, dielectric loss occurs due to their molecular motion and the loss of electrical energy is finally transformed into thermal energy. In our apparatus this dielectric loss is detected from the temperature difference between the sample in the electric field and the reference sample.

As seen in eq 1 , the quantity of the exothermic heat $Q$ per unit volume per $1 \mathrm{sec}$ depends on the dielectric loss $\varepsilon^{\prime \prime}$, frequency $f$, and on the intensity of the electric field $E_{0}$.

$$
Q=2 \pi f^{\prime \prime} E_{0}{ }^{2} / 8 \pi=\varepsilon^{\prime \prime} f E_{0}{ }^{2} / 4
$$

The important assumption on which the DTA method is based is that the temperature difference $\Delta T$ between sample and reference materials (the same size and shape as sample side) is proportional to the exothermic heat quantity $Q$. Then the following relation is obtained:

$$
\Delta T \propto \varepsilon^{\prime \prime} f E_{0}{ }^{2}
$$

If the thickness of the sample is taken to be uniform throughout the measuring process, $\varepsilon^{\prime \prime}$ may be expressed by the following equation:

$$
\varepsilon^{\prime \prime}=A \Delta T / f V_{0}^{2}
$$

where $V_{0}$ is the applied voltage and $A$ is a proportionality constant related to the density and specific heat at constant pressure of the sample.

If the height from the base line $(\Delta h)$ in an obtained DTA thermo-gram is used as a substitute for $\Delta T$ and the measurement carried out under constant voltage and frequency, the following equation is obtained:

$$
\varepsilon^{\prime \prime *}=B \varepsilon=\Delta h / f V_{0}^{2}
$$

where $B$ is a proportionality constant related to the distance between sample and thermocouple and the sensitivity of recorder, and $\varepsilon^{\prime \prime *}$ is a quantity directly related to $\varepsilon^{\prime \prime}$. When the applied voltage $V_{0}$, frequency $f$, thickness of sample, and proportionality constant between $Q$ and $\Delta h$ are all known, $\varepsilon^{\prime \prime}$ is easily calculated from $\varepsilon^{\prime \prime *}$.

\section{EXPERIMENTAL}

\section{Materials}

Poly(vinyl nitrate) (PVN) was synthesised from poly(vinyl alcohol) according to our previous publication $^{4,7}$. The degree of nitration was 86.0 mol\% with a Degree of Polymerization (DP) of 1700. Poly(vinyl acetate) (PVAc) was a commercial product with a DP of 1800 . Ethylene vinyl acetate copolymer (EVAc) was a product furnished by Nippon Gosei Kagaku Co., Ltd., with a DP of 3000 . All of these polymers were purified by reprecipitation from acetone solutions with distilled water several times. A random copolymer, poly(VC-VAc) (VC:VAc, 5:5), and a graft copolymer, (PVC-EVAc) graftcopolymer (PVC: EVAc, $7: 3 ; 45$ wt $\%$ of VAc in EVAc), were supplied from the Japanese Geon Company, Ltd.

Polymer mixtures were prepared from mixed solutions of polymer pairs by evaporating the solvent acetone. All casting films were dried at $40-50^{\circ} \mathrm{C}$ in vacuo for more than two weeks until constant weights were reached. The superposed sample was made by combining two polymers (not blend) PVN : EVAc, PVN : PVAc, (both about $3: 7$ ) in the holder shown in Figure 4.

\section{Apparatus}

Block diagrams of the aparatus are shown in Figure 1, and the main parts of the apparatus for high (above room temperature measurements are shown in Figures 2 and 3.

A piece of testing sample was prepared by hot-pressing the polymer film between aluminum foils (thickness of the obtained films were about $0.05 \mathrm{~mm}$ ); it was cut to a constant shape and size as shown in Figure 4. In this type of sample holder, the exothermic contribution due to the surface current must be taken into consideration. However, it was confirmed to be negligibly small with a blank test.

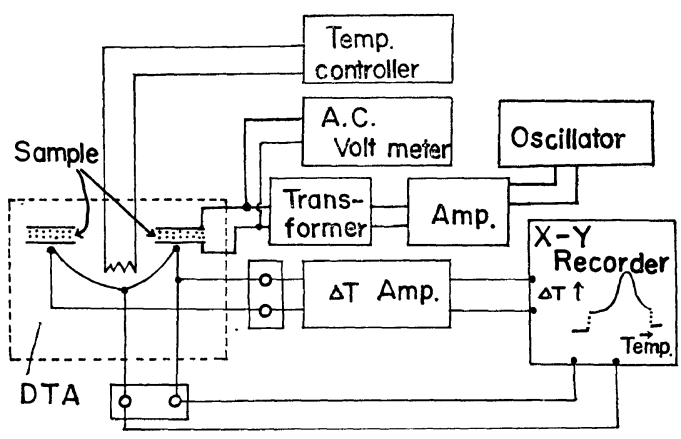

Figure 1. Block diagram of the apparatus for dielectric loss measurement. 


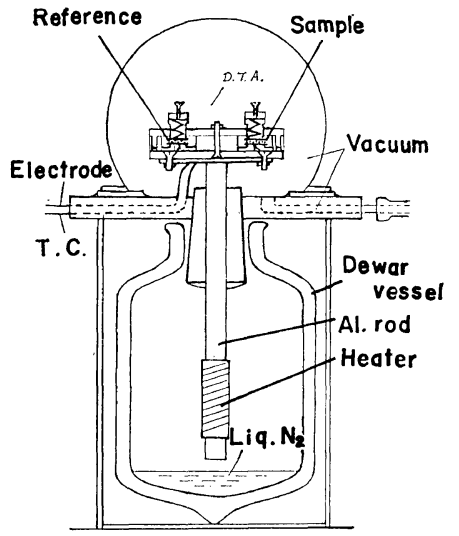

Figure 2. Thermo-dielectric loss measurement apparatus for low temperature.

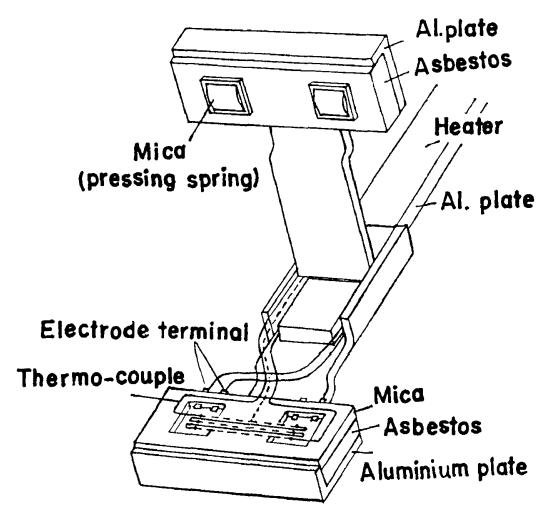

Figure 3. Thermo-dielectric loss measurement apparatus for high temperature.

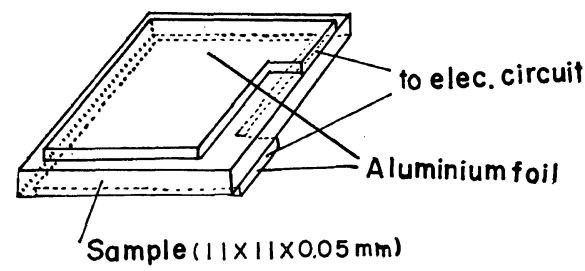

Figure 4. Shape and size of the test sample.

The frequency range was from $100 \mathrm{kHz}$ to 500 $\mathrm{kHz}$. The applied electric field was about 10 $\mathrm{kV} / \mathrm{cm}$ and the temperature elevation rate was $1-2{ }^{\circ} \mathrm{C} / \mathrm{min}$.

In the low temperature apparatus, sample holders were covered with a glass ball, as shown in Figure 2. The atmosphere in this ball was replaced by low pressure dried nitrogen gas before cooling the aluminum rod, to prevent the condensation of water vapor.
Measuring Method and Estimation of Compatibility

When the electric field is applied, the DTA curves rise quickly from their base lines; after turning off the field, they return rapidly to the initial base lines, as shown in Figure 5. The $\Delta h$ in eq 4 is the height of the DTA curve from the base line (1) in Figure 5.

From the DTA curves at various frequencies, $\varepsilon^{\prime \prime *} \sim T$ curves of polymers can be obtained by calculations using eq 4 of the theory; these curves are shown in Figures 6 and 7, for various samples. From these curves the contour maps of dielectric loss can be drawn as well, for example, as shown in Figure 8; $\log f$ can be plotted against $1 / T$ taking the values of $\varepsilon^{\prime \prime *}$ perpendicular to the paper surface. One of the advantages of this method is that drawing these contour maps is performed easily, because the data at various frequencies can be obtained in a very short time as compared with the usual purely electrical method; the amount of the sample required for the measurement is also small.

As shown in these contour maps, the determination of polymer compatibility is possible from the characteristic difference between compatible and incompatible mixtures, for the former shows a single mountain chain line, while the latter shows these lines as doubled. This distinction is based on the same considerations as that of the glass transition behavior between compatible and incompatible mixtures.

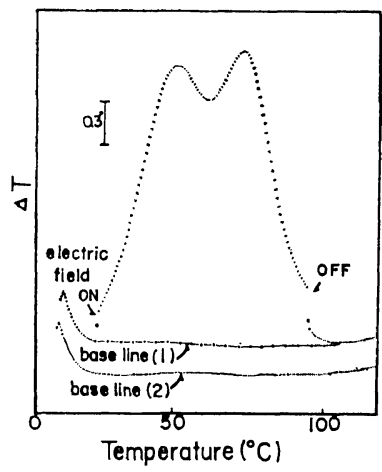

Figure 5. DTA curve of dielectric loss for an incompatible mixture (PVAc-EVAc, at $10 \mathrm{KHz}$ ). -.-, the base line (1) drawn parallel to base line $(2) ; \cdots$, , the base line (2) which is obtained by putting the same samples into both sample and reference sides of holders with no electric field applied. 
Activation energies of $\alpha$-relaxation (WLF type) of each polymer and compatible mixture are determined from the slopes of the mountain chains in those maps.

The curves of normalized dielectric loss at definite frequency $v s$. temperature are shown in Figure 18 , where $\varepsilon_{\max }^{\prime \prime}$ is the maximum value of $\varepsilon^{\prime \prime *}$ and $T_{E \max }$ is the temperature at which the dielectric loss becomes maximum. The same curves at constant temperature, $v s$. frequency are shown in Figure 19, where $f_{\max }$ is the frequency at which the dielectric loss becomes maximum. In these Figures, the shapes of the temperature or frequency dispersion curves of polymers and their compatible mixtures are clearly different. Then, an approximate estimation of polymer compatibility may be presumed by a comparison of the sharpness of these curves.

\section{RESULTS AND DISCUSSION}

The curves of $\varepsilon^{\prime \prime *}$ at various frequencies are shown in Figure 6 for PVAc. At the same time,

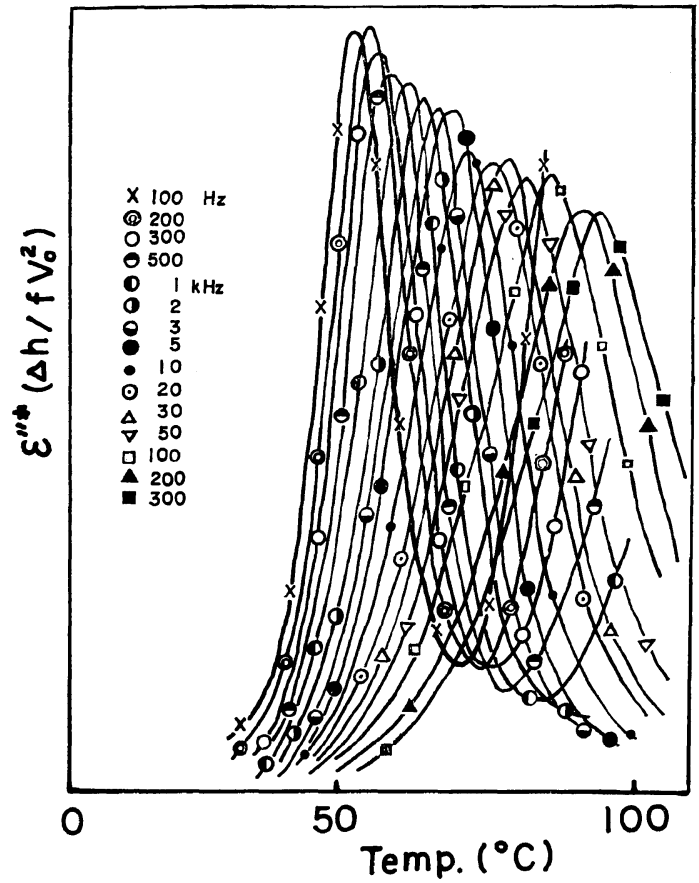

Figure 6. Temperature dispersion of $\varepsilon^{\prime *}$ at various frequencies for poly(vinyl acetate) (PVAc). The symbols indicate the written frequencies respectively, and the same symbols are used in the next Figure 7.

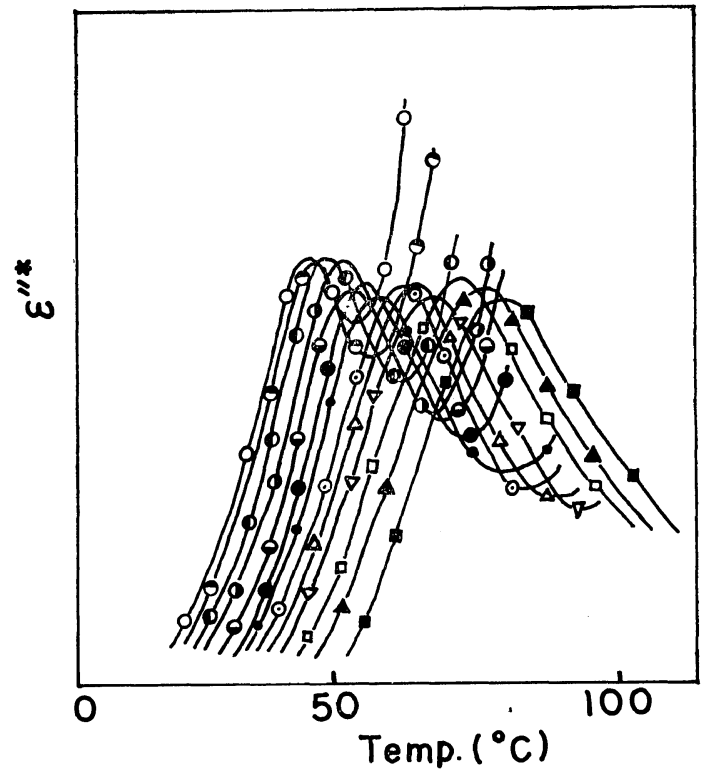

Figure 7. Temperature dispersion of $\varepsilon^{\prime \prime *}$ at various frequencies for a compatible mixture (PVN : EVAc, $3: 7$, wt ratio).

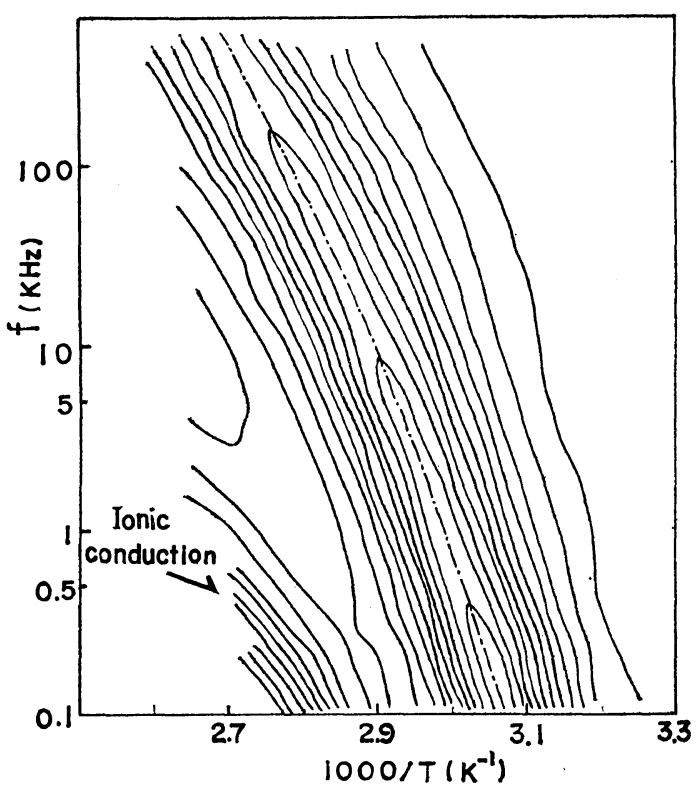

Figure 8. Contour map of $\varepsilon^{\prime \prime *}$ for PVAc. - - represents a mountain chain and this is the same in the other maps.

typical curves for compatible mixtures are shown in Figure 7. In the case of incompatible mixtures, double peaks were observed in the every 


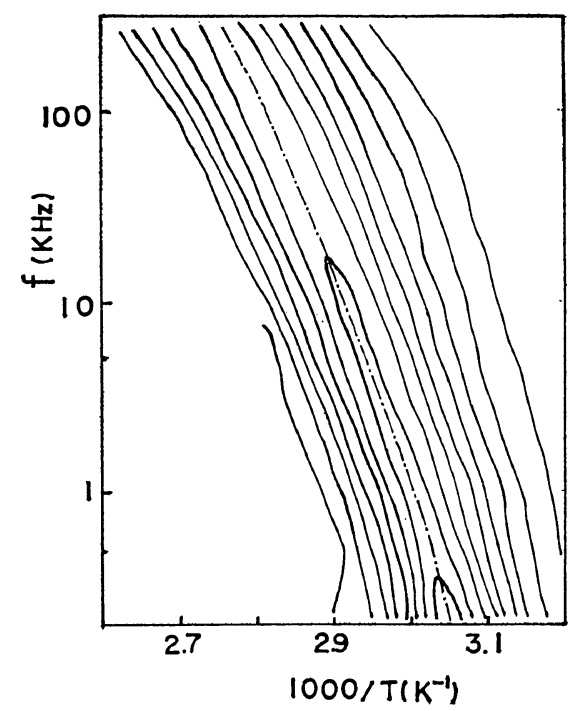

Figure 9. Contour map of $\varepsilon^{\prime \prime}$ for PVN.

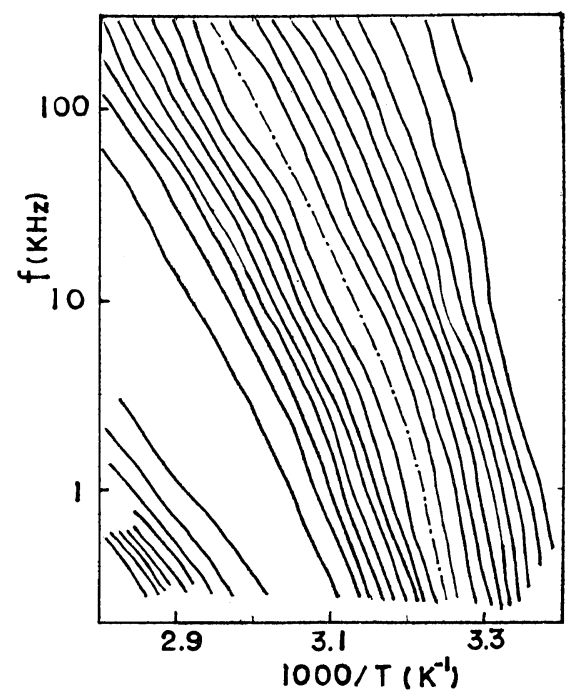

Figure 10. Contour map of $\varepsilon^{\prime \prime *}$ for EVAc.

composition of mixing, even those not shown here (PVAc:EVAc, 3:7, 4:6, 5:5, 7:3, wt ratio), while a single peak was observed in the compatible case (PVN : PVAc, 1:9, 3:7, 5:5, $7: 3$, and $9: 1$; PVN : EVAc, $1: 9,3: 7,5: 5$, $9: 1$, wt ratio). In these curves, the rise of $\varepsilon^{\prime \prime *}$ at high temperature which is caused by the ionic conduction were observed, as can be partly seen in Figure 6.

The contour maps of three polymers are shown

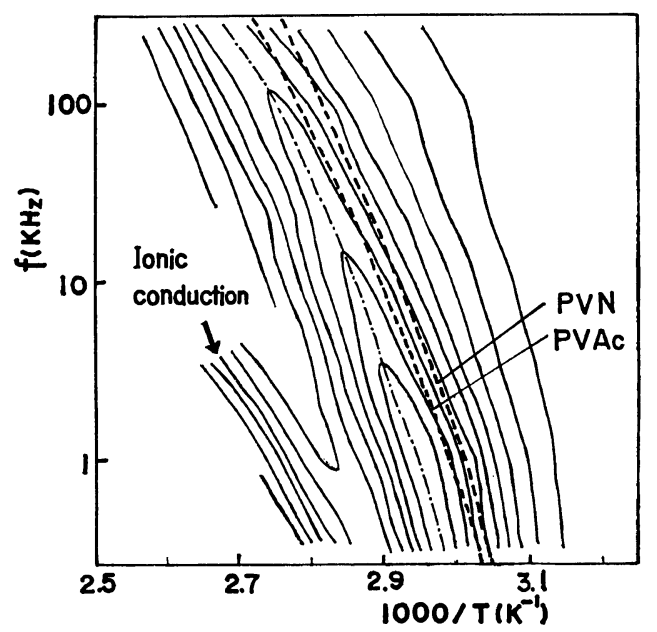

Figure 11. Contour map of $\varepsilon^{\prime *}$ for the compatible mixture (PVN: PVAc, $3: 7$ ). The dotted lines represent the mountain chains of PVN and PVAc, respectively.

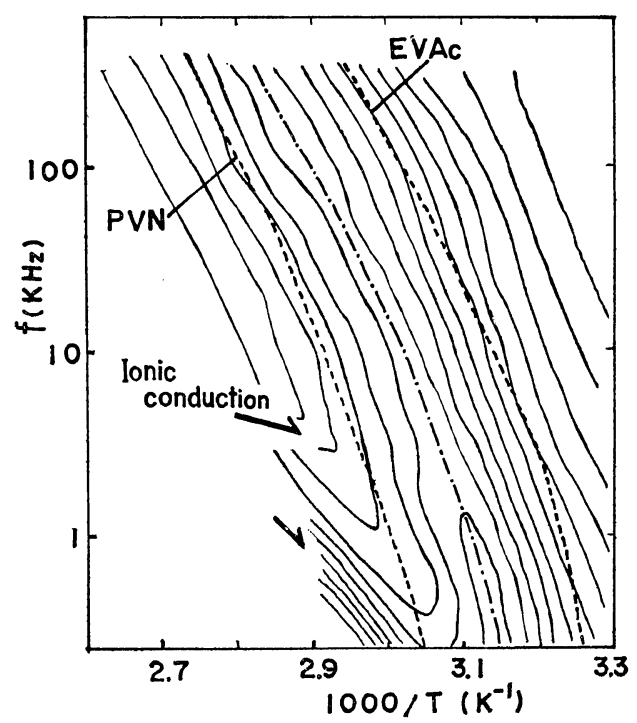

Figure 12. Contour map of $\varepsilon^{\prime \prime *}$ for the compatible mixture (PVN : EVAc, $3: 7$ ). The dotted lines represent the mountain chains of PVN and EVAc, respectively.

in Figures 8, 9, and 10 for PVAc, PVN, and EVAc, respectively. The compatible mixtures are shown in Figures 11 and 12, where single mountain chains are observed in both maps. The incompatible mixture is shown in Figure 13, where incontrast double maintain chains are ob- 


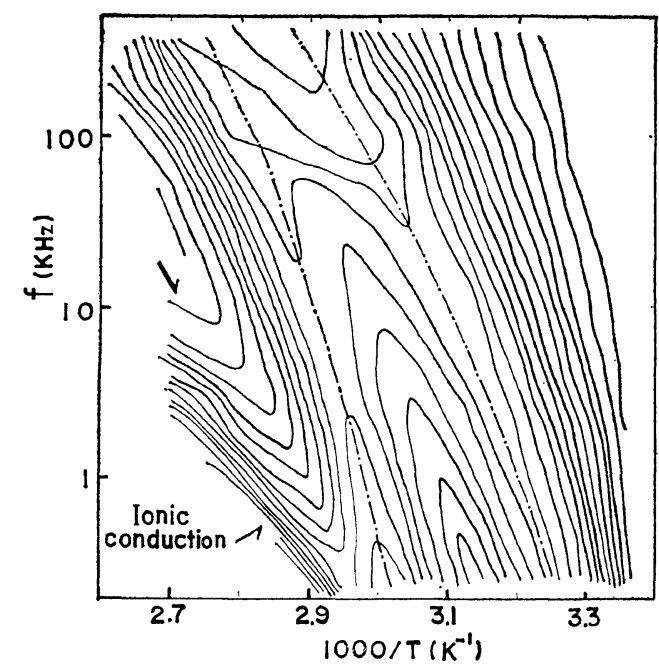

Figure 13. Contour map of $\varepsilon^{\prime \prime *}$ for the incompatible mixture (PVAc: EVAc, $4: 6$ ).

served. In the case of PVN-PVAc mixtures, the position of single mountain chains are shifted to higher temperatures than those of either component polymers (the dotted lines), as represented in Figure 11. In the case of PVN-EVAc mixtures, the single mountain chains appear in the middle position between those of both component polymers, as also seen for example, in Figure 12. But in the case of PVN-EVAc superposed sample, the double mountain chains were observed clearly, although not shown here. These results provide strong evidence that the appearance of single mountain chains is due to the compatible PVN-EVAc mixtures, and not to the closed peaks resulting from the low re-

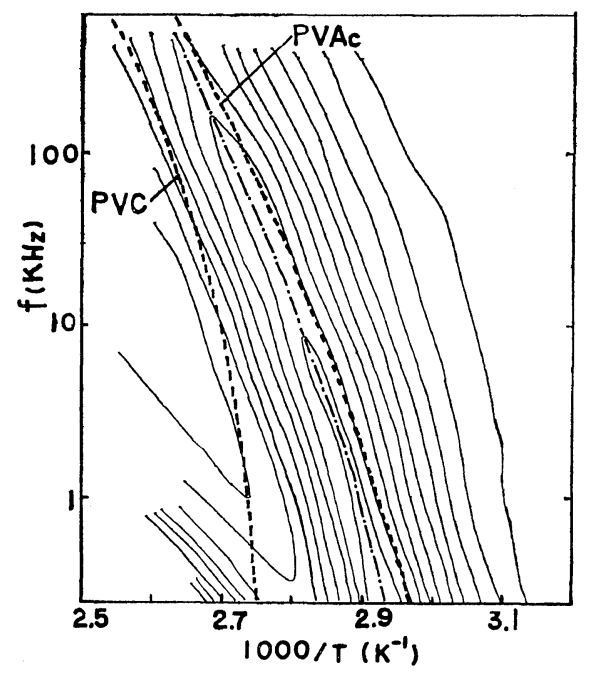

Figure 14. Contour map of $\varepsilon^{\prime \prime *}$ for random copolymer poly(VC-Co-VAc) (VAc, $50 \mathrm{wt} \%)$. The dotted line represent the mountain chains of PVAc and PVC, respectively.

solution of this apparatus. Also, in the case of PVN_PVAc superposed sample, the single mountain chain was not shifted from the positions of component polymers. The position of superposed sample appeared between those of PVN and PVAc, although not shown here. This fact supports the existence of interactions in the PVN_PVAc mixture.

The map of a random copolymer is shown in Figure 14. Since this map shows a single mountain chain between those of both component homopolymers, the shape of the map for a compatible mixture (PVN-EVAc) is considered

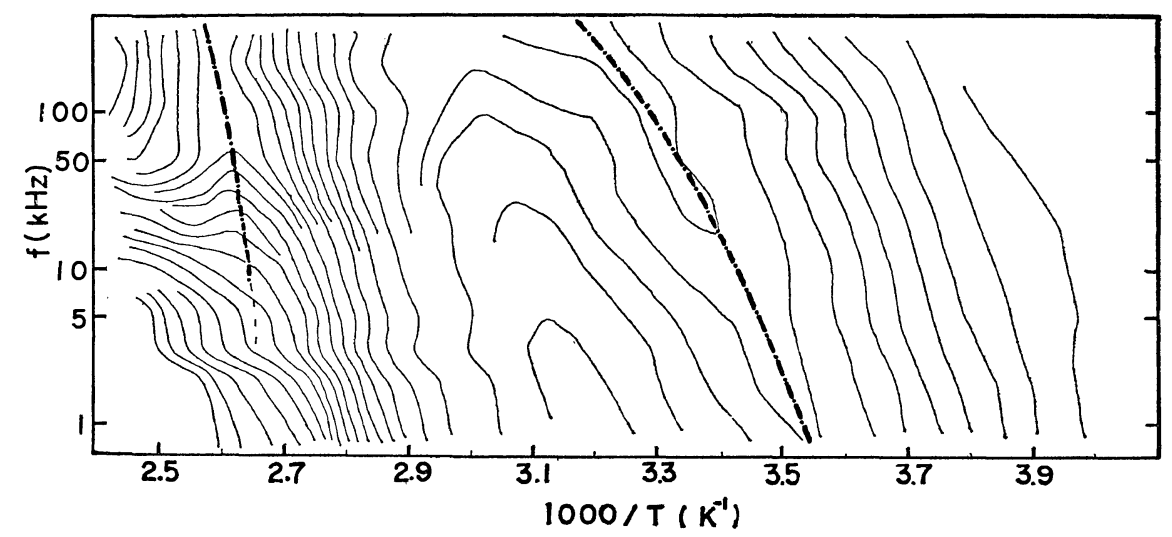

Figure 15. Contour map of $\varepsilon^{\prime \prime *}$ for graft copolymer, (PVC-VAc) graftmer (VAc, $\left.30 \mathrm{wt} \%\right)$. 
to be very similar to that of random copolymer. In addition, the map of a graftmer is shown in Figure 15, for comparison with that of an incompatible mixture (Figure 13). Since the double mountain chains are obviously observed in this map, the shape for incompatible mixture is also considered to very similar to that of a graftmer. These double mountain chains may be expected, because the graftmer is composed of two different polymer phases.

Activation energies of dielectric relaxation pro-

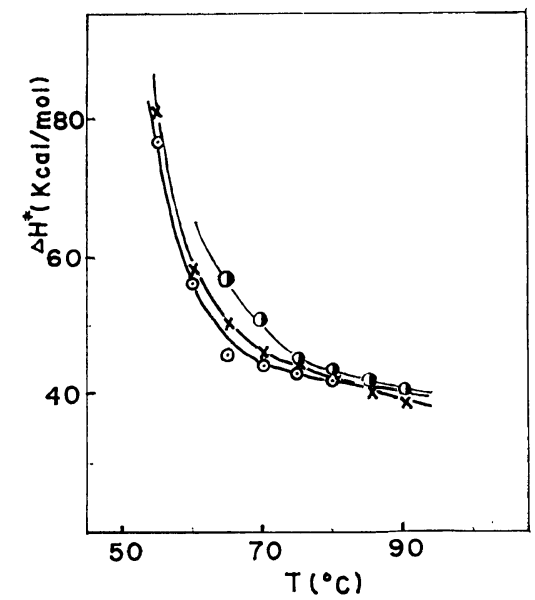

Figure 16. Temperature dependence of activation energy for the compatible mixture (PVN-PVAc): $\odot$, PVN; $\times$, PVAc; $1, \operatorname{PVN}: \operatorname{PVAc}(3: 7)$.

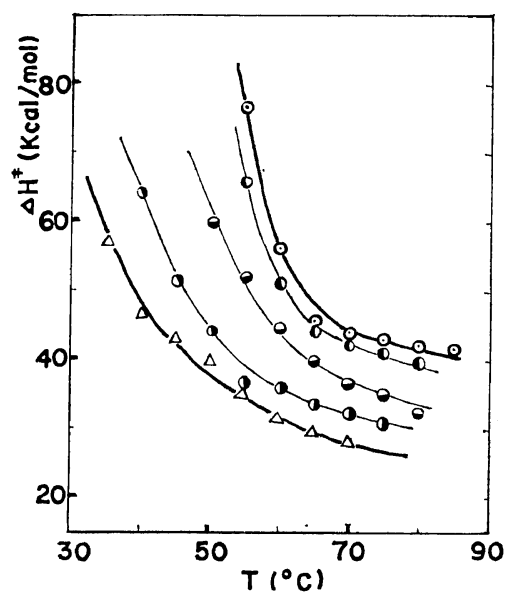

Figure 17. Temperature dependence of activation energy for the compatible mixture (PVN-EVAc): $\odot$, PVN; $\triangle$, EVAc; D, PVN : EVAc (5:5); $\ominus$, PVN : EVAc (3:7); P, PVN : EVAc (1:9). cess of the segments in the compatible mixtures are shown in Figures 16 and 17, for PVN-PVAc and PVN-EVAc mixtures, respectively. The values of activation energy $\left(\Delta H^{\ddagger}\right)$ of $\mathrm{PVN}-$ PVAc are found to be larger than those of component polymers, as seen in Figure 16. It can be seen from this figure that the values of PVN and PVAc are nearly equal to each other. The larger $\Delta H^{\ddagger}$ of the mixture may also indicate the existence of a special attractive interaction between segments of PVN and of PVAc.

In previous reports, ${ }^{4,5}$ we have discussed the existence of this characteristic intermolecular interaction. On the other hand, the $\Delta H^{\neq}$values
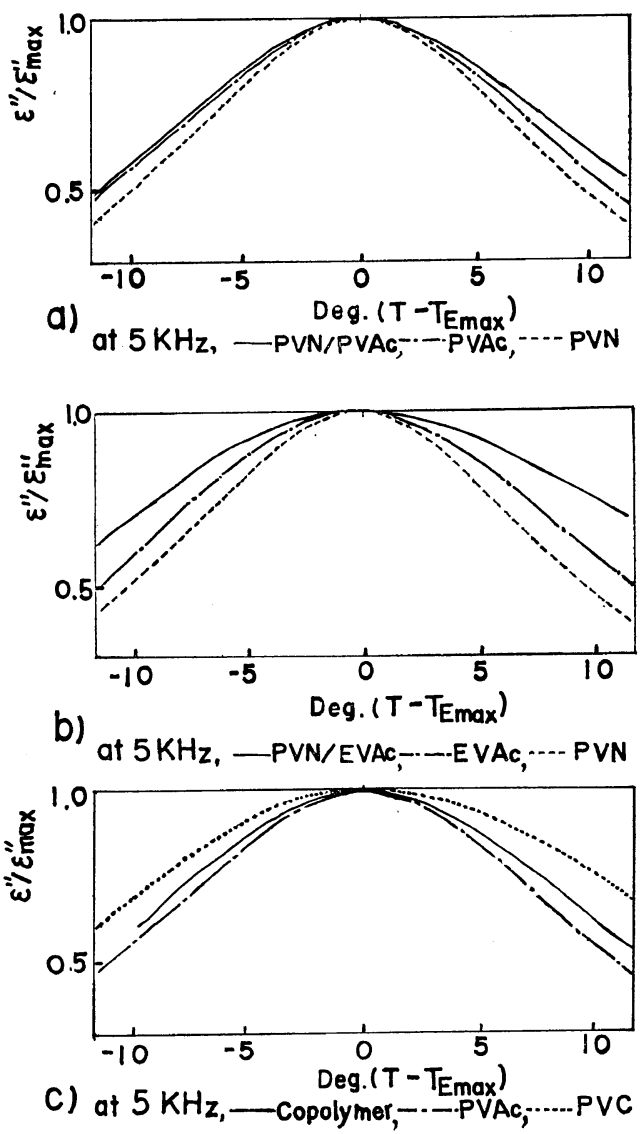

Figure 18. The sharpness of temperature dispersion curves of normalized dielectric loss for the compatible mixtures and a random copolymer at constant frequency: (a), PVN-PVAc-(3:7) mixture; (b), PVN-EVAc-(3:7) mixture; (c), poly(VC-Co-VAc); $T_{E \max }$, temp. of dielectric loss become maximum; $\varepsilon_{\max }^{\prime \prime}$, maximum value of $\varepsilon^{\prime \prime}$. 
of PVN-EVAc mixture lie in between those of component polymers, as seen in Figure 17.

In Figure 18, the normalized dielectric loss curves of compatible mixtures and of a random copolymer are shown at a constant frequency. It is found that the estimation of homogeneity may also be determined by the sharpness of these curves. Comparing the sharpness of curve a) with curve b) in Figure 18, we considered that the PVN_PVAc mixture, may be more homogeneous than the PVN-EVAc mixture because the former is more sharp than the latter curve. The ratio obtained from the halfwidths of the two mixtures is 1.28 at $5 \mathrm{kHz}$. It is also found that the sharpness of the curve for the random copolymer lies in the middle position between the two component polymers, as seen by c) in this Figure, while in the case of compatible mixtures this sharpness is less pronounced than in either component, as also seen in a) and b).

In Figure 19, the normalized dielectric loss curves of the above mixture are also shown at constant temperature. In these frequency dispersion curves, the PVN-PVAc mixture seemed to be more sharp than PVN-EVAc mixture,

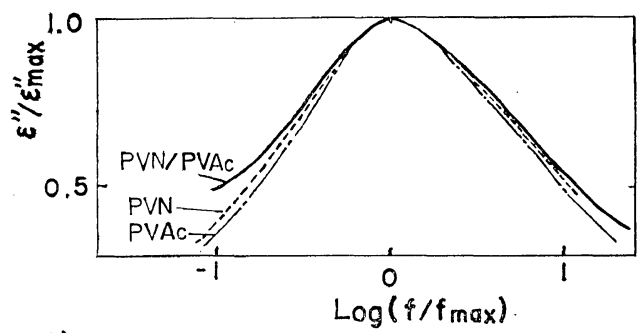

a) at $70^{\circ} \mathrm{C}$

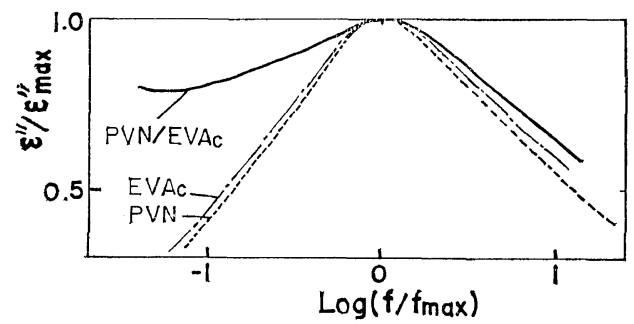

b) a† $60^{\circ} \mathrm{C}$

Figure 19. The sharpness of frequency dispersion curves of normalized dielectric loss for the compatible mixtures at constant temperature: $f_{\max }$, the frequency of $\varepsilon_{\max }^{\prime \prime *} ;($ a), PVN-PVAc-(3:7) mixture, (b), PVN-EVAc-(3:7) mixture. though there is an unavoidable temperature difference of $10^{\circ} \mathrm{C}$ between the two cases. Then, the same result is assumed for the difference of compatibility between the two compatible mixtures. And from the broadness of the distribution curves of relaxation times, it is also clear that the mixture curves are more widely spread than their component curves as seen by a) and b). The temperatures are so taken from their contour maps that the single peaks of frequency dispersion curves appeared clearly in each cases with cutting off the effect of dielectric conduction and with little temperature difference. Therefore, the adequate temperatures are limited in these cases. The ratio obtained from the halfwidths of the two mixtures is 1.27 at the given temperature.

The glass transitions $\left(T_{\mathrm{g}}\right)$ obtained by DTA method are shown in Figure 20. The $T_{\mathrm{g}}$ values of PVN-PVAc mixture agree closely with the values from a modified Gordon-Taylor expression obtained in a previous study. ${ }^{5}$ The values of the PVN-EVAc mixture indicate that the same application will be possible to these values.

In Figure 21, $T_{E \max }$ value are shown at various frequencies. These value of PVN-EVAc mixture $v s$. composition are seen to be slightly higher than the component polymer values in the middle compositions. And the values of PVN-EVAc mixture vs. composition are also found to have a convex shape. Therefore, $T_{E \max }$ of the compatible mixtures at various frequencies correspond well with their $T_{\mathrm{g}}$ values obtained by the DTA

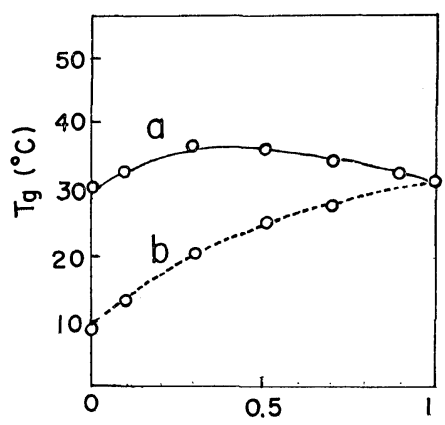

Wit fraction of PVN

Figure 20. Glass transition temperatures $\left(T_{\mathrm{g}}\right)$ of the compatible mixture obtained by DTA method; (a), PVN-PVAc series, the full line represents the modified Gorden-Taylor expression ${ }^{5}$; (b) PVNEVAc series. 

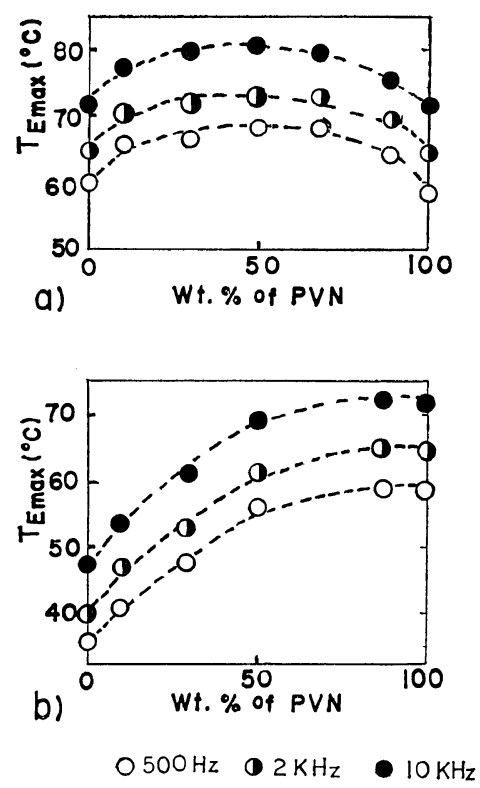

Figure 21. The temperatures at which dielectric loss become maximum $\left(T_{E \max }\right) v s$. compositions of the compatible mixtures at various frequencies: (a), PVN-PVAc series; (b), PVN-EVAc series; $\bigcirc, 500 \mathrm{~Hz} ; 0,2 \mathrm{kHz} ; \bigcirc, 10 \mathrm{kHz}$.

method. These facts strongly indicate the existence of intermolecular interaction between the polar polymers. And our previous results ${ }^{4,5}$ were also supported by these experiments. This compatibility will be established by their interaction.

From the above results, a new method for estimation of polymer compatibility is found to be possible using the characteristic appearances of the contour maps. It is also found that even among compatible mixtures, the difference of homogeneity may be estimated by the frequency or temperature dispersion curves of normalized dielectric loss.

Recently, we obtained other results which showed that this estimation method is more sensitive than the dynamic mechanical method for investigating the aggregation state of polymer mixtures, ${ }^{8}$ and also that by precise thermal measurements the heat of mixing is exothermic ${ }^{9}$ for both PVN-PVAc and PVN-EVAc mixtures at $30^{\circ} \mathrm{C}$; thus the compatibility of these mixture is confirmed thermodynamically.

More detailed information about the compatibility of polymer mixtures or state of aggregation of polymers will be found by the further extension of the frequency and temperature ranges of the measurement and also by making these contour maps more precise.

Acknowledgment. Part of this work was supported by the Grant-in-Aid for Experimental Research (885166) from the Ministry of Education, Japan.

\section{REFERENCES}

1. T. Matsuo, H. Suga, and S. Seki, Bull. Chem. Soc. Japan, 39, 1827 (1966); J. Phys. Soc. Japan, 22, 677 (1967).

2. K. Amaya, K. Sasaki, and T. Tanaka, Abstract, 3th Symposium of Calorimetry Conference, Osaka, Japan, Nov., 1967, p 110.

3. R. Kaneko, "Calorimetry, Thermometry and Thermal Analysis", Vol. 6, edited by Society of Calorimetry and Thermal Analysis, 1973, p 10.

4. S. Akiyama, N. Inaba, and R. Kaneko, Kobunshi Kagaku (Chem. High Polymers), 26, 529 (1969).

5. S. Akiyama, Bull. Chem. Soc. Japan, 45, 1381 (1972).

6. S. Akiyama and R. Kaneko, Kobunshi Ronbunshu, 31, 12 (1974).

7. S. Akiyama and R. Kaneko, Kobunshi Kagaku (Chem. High. Polymers), 25, 145 (1968).

8. Y. Komatsu, S. Akiyama, and R. Kaneko, Abstract, SPSJ 23th Annual Meeting, Tokyo, Japan, June, 1974, p 375.

9. S. Akiyama, R. Kaneko, and Y. Komatsu, Abstract, SPSJ 23th Annual Meeting, Tokyo, Japan, June, 1974, p 504. 